\title{
An Error Analysis for the Measurement of Satellite EIRP Using a Calibrated Radio Star
}

\author{
WILLIAM C. DAYWITT
}

\begin{abstract}
An outline for the derivation of equations employed in a measurement and error analysis of satellite EIRP using a calibrated radio star is presented. A table showing analysis results at $7.55 \mathrm{GHz}$ using Cassiopeia $A$ for a satellite at $12^{\circ}$ elevation angle is given. The quadrature sum of the systematic errors appearing in the table is $\mathbf{1 0 . 1}$ percent. Also presented is a curve of the systematic errors as a function of elevation angle showing a 7.3-percent minimum at high elevation angles.
\end{abstract}

\section{INTRODUCTION}

$\mathrm{T}$ HE NATIONAL Bureau of Standards has constructed a system designed to measure satellite EIRP using a calibrated radio star. The system operates off the IF patch panel of the downlink earth terminal (ET), and automatically controls the several measurement sequences.

The method employed in the earth-terminal measurement system (ETMS) is a modified version of the radio-star method [1] of measuring satellite EIRP (effective isotropic radiated power), the modification [2] allowing ET receiver gain fluctuations to be removed from the measurement and significantly reducing measurement error.

In addition to a digital voltmeter and accurately calibrated IF attenuators, the ETMS features a very accurate power bridge [3], and an improved solid-state noise source [4] of high stability. Combined, the components can routinely measure power ratios to a few hundredths of a decibel. The ETMS also incorporates well calibrated IF bandpass filters whose characteristics are combined with the observed ET passband characteristics by the computer software in the ETMS to obtain the overall noise bandwidth needed for the calculation of EIRP.

A measurement and error analysis for the $1-10-\mathrm{GHz}$ frequency range to be reviewed in this paper is incorporated in the ETMS computer software. The analysis reveals additional errors and a higher atmospheric correction than previously calculated.

The relationship of the ETMS to the ET receiving system is shown in Fig. 1. The receiving system is represented by the ET antenna, cross-guide coupler, low-noise amplifier (LNA), downconverter, and IF patch panel. The ETMS consists of the solid-state noise source, and the power measurement and control console. The noise source is connected to the receiving system through the cross-guide

Manuscript received December 2, 1977. This work was supported in part by the U.S. Army Communications Command, Fort Huachuca, AZ. The author is with the U.S. Department of Commerce, National Bureau of Standards, Boulder, CO 80302.

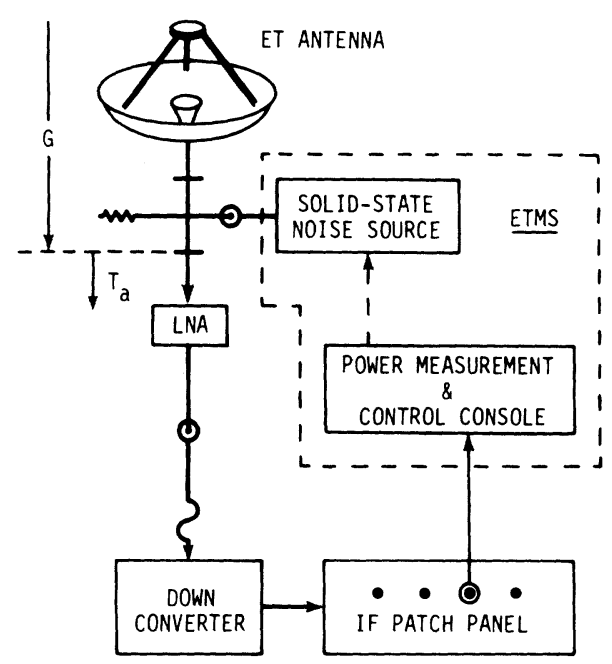

Fig. 1. Interface between the ETMS and the earth-terminal receiving system.

coupler, and $T_{a}$ is the noise temperature representing the noise power added to the system at the LNA when the noise source is turned on. The gain of the system from the antenna far field to the LNA is represented by $G$, and is the product of the antenna gain and the waveguide loss from the antenna to the LNA.

The noise source serves a double purpose in the EIRP measurement. The first is to eliminate gain fluctuations [2] from the LNA input to the power detector in the ETMS. This is accomplished by performing all power measurements in pairs, one measurement with the noise source turned off, followed immediately by the same measurement with the noise source turned on. The noise-source-off measurement is then normalized by the difference between the two measurements, thus eliminating the system gain (and consequently the possibility of gain fluctuations) between the LNA and the ETMS power detector from the power ratio. Besides the immediate benefit of removing these short-term gain fluctuations, measurements may now be compared accurately on a day-to-day basis. The second purpose is to provide a calibratable source of power by which the magnitude of the received satellite signal power can be measured. The calibration of this noise power is accomplished in the first step of the EIRP measurement by comparing the reduced noise temperature $\left(T_{a} / G\right)$ of the noise source against the power from a calibrated radio star.

The EIRP measurement method employed by the ETMS involves two steps: 1) a radio-star calibration of the reduced 


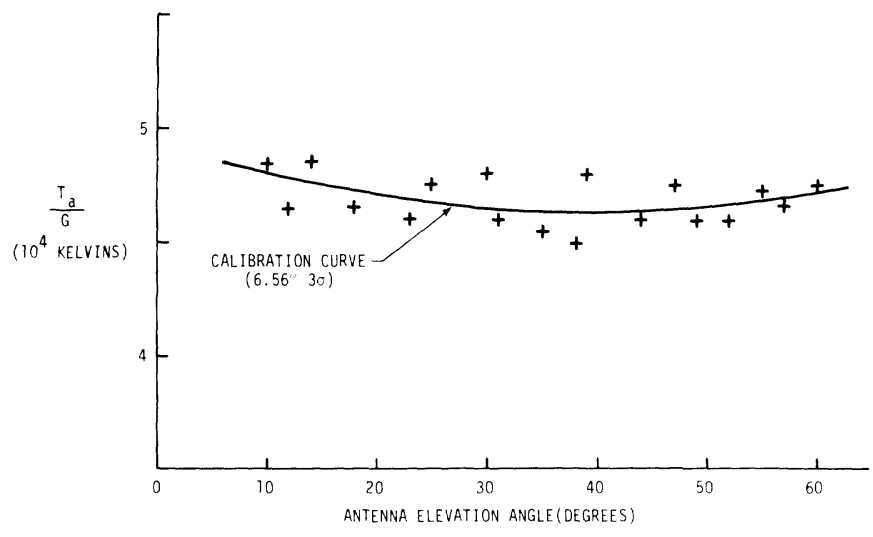

Fig. 2. $T_{a} / G$ ET calibration curve.

noise temperature $\left(T_{a} / G\right)$ of the solid-state noise source as a function of ET antenna elevation angle, and 2) the measurement of the available powers at the LNA input received from the satellite. The first step is performed by measuring the IF power from the ET receiving system as the radio star drifts through the mainbeam of the antenna. These measurements are performed at various elevation angles as the star traverses its path across the sky, and yield a set of $T_{a} / G$ data points as depicted in Fig. 2. The least squares curve fit to these data points represents a calibration curve for the "front end" of the receiving system, and the solid-state noise source. The 6.5-percent value appearing in the figure represents random scatter $(3 \sigma)$ of the data points. The curve remains valid as long as $T_{a}$ and $G$ remain constant in time. Measurements performed on the noise source [4] show it to be stable to within $\pm 0.01 \mathrm{~dB}(1 \sigma)$ over at least the time required to complete a set of EIRP measurements for a number of frequencies. The gain $G$ is assumed to remain constant for this same period, an assumption that appears to be born out by experience. Once the calibration curve is established, the value of $T_{a} / G$ for any satellite elevation angle can be easily determined by reading the value of $T_{a} / G$ from the curve at that angle.

After obtaining the $T_{a} / G$ calibration curve, the ET antenna is pointed at the satellite whose EIRP is to be measured, and a number of measurements of received satellite power are performed. The average of these measurements normalized by $T_{a}$ yields a $\Delta Y$ that can be used with the proper (at the satellite elevation angle) $T_{a} / G$ value to determine the satellite EIRP from the equation

$$
\operatorname{EIRP}=\frac{k\left(T_{a} / G\right) \Delta f L \Delta Y}{A e_{1} \cdots e_{7}}
$$

where $k$ is Boltzmann's constant, $\Delta f$ is the combined ET-ETMS noise bandwidth, $L$ is the space loss $(4 \pi r / \lambda)^{2}[5]$, $r$ is the range from the ET antenna to the satellite transmit antenna, $\lambda$ is the measurement wavelength, and $A$ is the aspect angle correction [1]. $e_{1}$ through $e_{7}$ are near-unity correction factors analogous to the $G / T$ correction factors described in an earlier publication [6]. Sequentially, these factors account for: 1) atmospheric transmission losses, 2) the angular extent of the received satellite signal as "seen" by

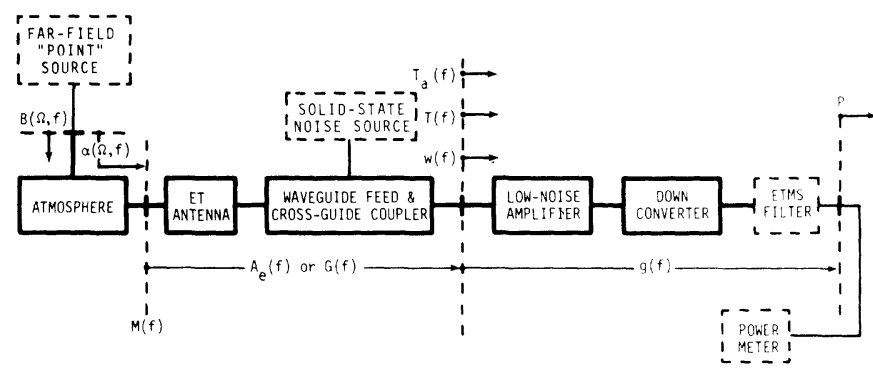

Fig. 3. Block diagram of a model representing the ET receiving system.

the ET antenna, 3) frequency variations of the receiving system components across the receiver passband, 4) frequency variations of the downlink operating noise temperature across the receiver passband, 5) imperfect ET antenna pointing, 6) polarization mismatch between satellite signal and ET antenna, and 7) finite response time of the ETMS and instabilities in $T_{a}$.

The theoretical development leading to (1) is outlined in the next section.

\section{Theoretical Development}

As mentioned earlier, the EIRP measurement performed by the ETMS is accomplished in two steps, obtaining the $T_{a} / G$ calibration curve, and measuring the received satellite power. Before examining these two steps separately, basic equations used as a starting point for their theoretical descriptions will be presented. Although these equations and some of the approximations leading to the final working equations may be applicable over a wider frequency range, they are designed primarily for the $1-10-\mathrm{GHz}$ frequency range.

A model of the ET receiving system is shown in the block diagram of Fig. 3. The system (including the atmosphere) is outlined in solid blocks. The far-field source is the satellite or radio star, depending at which the ET antenna is pointed, and $B(\Omega, f)$ is the corresponding brightness distribution [7]. $\Omega$ represents the pair of spherical polar angles with origin at the ET antenna and $z$ axis along the center of the mainbeam, and $f$ is the frequency. $\alpha(\Omega, f)$ is an operator representing the effects [8], [6] of gaseous absorption, refractive spreading, and tropospheric scattering on the incoming flux from the radio star or satellite. $M(f)$ is a mismatch factor [9] relating the polarization of the source radiation to the ET antenna polarization. $A_{e}(f)$ and $G(f)$ are the effective area and gain, respectively, of the combined antenna, waveguide feed, and cross-guide coupler. $T_{a}(f)$ is the noise temperature of the solid-state noise source as it appears at the LNA input. When the noise source is turned off the added noise $T_{a}(f)$ is zero. $T(f)$ is the noise temperature of the ET system and consists of cosmic background noise, atmospheric and man-made noise, ground noise, noise from $I^{2} R$ losses in the antenna, waveguide feed, and cross-guide coupler, the effective input noise temperature of the LNA, and radiated satellite noise when the ET antenna is pointed at the satellite. $w(f)$ is the spectral power (power per unit bandwidth) corresponding to the sum of the spectral power from the 


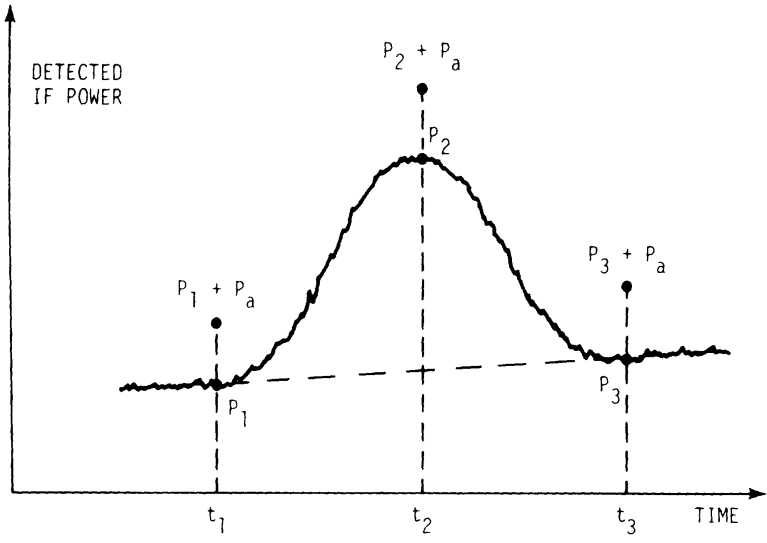

Fig. 4. IF power detected by the ETMS as a radio star traverses the ET antenna mainbeam.

far-field source $k T_{a}(f)$ and $k T(f) . g(f)$ is the ratio of the spectral power delivered to the power meter to the available spectral power at the LNA input. $P$ is the total power delivered to the power meter.

The spectral power $w(f)$ is related to other parameters appearing in Fig. 3 by the equation

$$
\begin{aligned}
& w(f)=k\left[T(f)+“ T_{a}(f) "\right]+A_{e}(f) M(f) \\
& \cdot \int \alpha(\Omega, f) B(\Omega, f) \cdot P_{n}\left(\Omega-\Omega_{0}, f\right) d \Omega .
\end{aligned}
$$

$P_{n}\left(\Omega-\Omega_{0}, f\right)$ is the normalized power pattern of the ET antenna, waveguide feed, cross-guide coupler combination, where the difference $\Omega-\Omega_{0}$ accounts for the fact that the antenna may not be pointing at the center $\Omega_{0}$ of the far-field source. The quotation marks around $T_{a}(f)$ are a reminder that $T_{a}(f)$ is zero when the solid-state noise source is turned off. $d \Omega$ is the infinitesimal solid angle $\sin \theta d \theta d \psi$.

The power $P$ detected by the ETMS is related to the spectral power $w(f)$ by the equation

$$
P=\int w(f) g(f) d f
$$

The form of (2) and (3) are a manifestation of the assumed linearity of the model in Fig. 3, and form the theoretical basis for the following description of the two-step measurement process leading to the EIRP equation (1).

\section{A. Step One- $T_{a} / G$ Calibration Curve}

The power measurements performed by the ETMS to obtain $T_{a} / G$ at a particular elevation angle are indicated by the heavy dots in Fig. 4. As a calibrated radio star passes through the center of the ET antenna mainbeam, the IF power detected by the ETMS describes a curve shaped somewhat as depicted in the figure. The slope of the baseline from $t_{1}$ to $t_{3}$ could be caused by a variation in the cosmic background surrounding the radio star, or a slow change in the ET receiver gain. Three pairs of power measurements are made, one at each time $t_{1}, t_{2}$, and $t_{3}$. At $t_{1}$, a measurement $P_{1}$ of the noise baseline is made, followed immediately by the same measurement $P_{1}+P_{a}$ with the solid-state noise source turned on. The ratio $y_{1}\left(=P_{1} / P_{a}\right)$ is then calculated to eliminate the receiver gain from consideration as previously described. Similar ratios $y_{2}\left(=P_{2} / P_{a}\right)$ and $y_{3}\left(=P_{3} / P_{a}\right)$ are calculated from measurements at $t_{2}$ and $t_{3}$. The measurement at $t_{2}$ corresponds to the radio star being in the center of the ET antenna pattern. The measurement at $t_{3}$ is another measurement of the noise baseline and which, with $y_{1}$, allows the value of the noise baseline at $t_{2}$ to be determined. By subtracting the noise baseline from the measurement at $t_{2}$, the IF power ratio difference $\Delta y$ corresponding to the radio star can be determined from the equation

$$
\Delta y=y_{2}-\left(y_{1}+y_{3}\right) / 2 .
$$

The values of the measured powers in Fig. 4 can be related to the quantities in the block diagram of Fig. 3 through (2) and (3). For example,

$$
\begin{aligned}
P_{2}=\int g(f)[k T(f)+ & A_{e}(f) M(f) \\
& \left.\cdot \int B(\Omega, f) \alpha(\Omega, f) P_{n}(\Omega, f) d \Omega\right] d f
\end{aligned}
$$

and

$$
P_{a}=\int g(f) k T_{a}(f) d f
$$

The $B(\Omega, f)$ in $(5)$ is the radio-star brightness distribution, and $M(f)$ (approximately equal to $1 / 2$ ) is the corresponding mismatch factor.

Equations (5) and (6) with similar equations for the measurements at $t_{2}$ and $t_{3}$ can be combined to give a theoretical expression for the $\Delta y$ of (4). When this is done and equated to $\Delta y$, the following equation can be derived:

$$
\frac{T_{a}}{G}=\frac{\lambda^{2} S}{8 \pi k} \cdot \frac{k_{1} \cdots k_{7}}{\Delta y} .
$$

$\lambda$ is the measurement wavelength, $S=B(\Omega, f) d \Omega$ is the radio-star flux density at frequency $f$, and $k$ is again Boltzmann's constant. $k_{1}$ through $k_{7}$ are correction factors defined to give the functional form of (7) and are expressions containing a number of integrals. Under certain circumstances, these factors are close to unity, making accurate radio-star measurement of $G / T$ or EIRP possible. Reduced expressions for the $k_{i}$ 's have been discussed in earlier publications [6], [12].

The value for $T_{a} / G$ calculated from (7) pertains to a particular elevation angle. When this process is repeated for other elevation angles as the radio-star traverses its path across the sky, the data points in Fig. 2 from which the calibration curve is derived are generated. The $T_{a} / G$ value needed to determine the satellite signal power is then obtained from the curve at the elevation angle of the satellite.

\section{B. Step Two-Received Satellite Power Measurements}

With the $T_{a} / G$ calibration completed, the antenna is pointed at the satellite in order to measure the received signal power. Fig. 5 illustrates the resulting RF power 


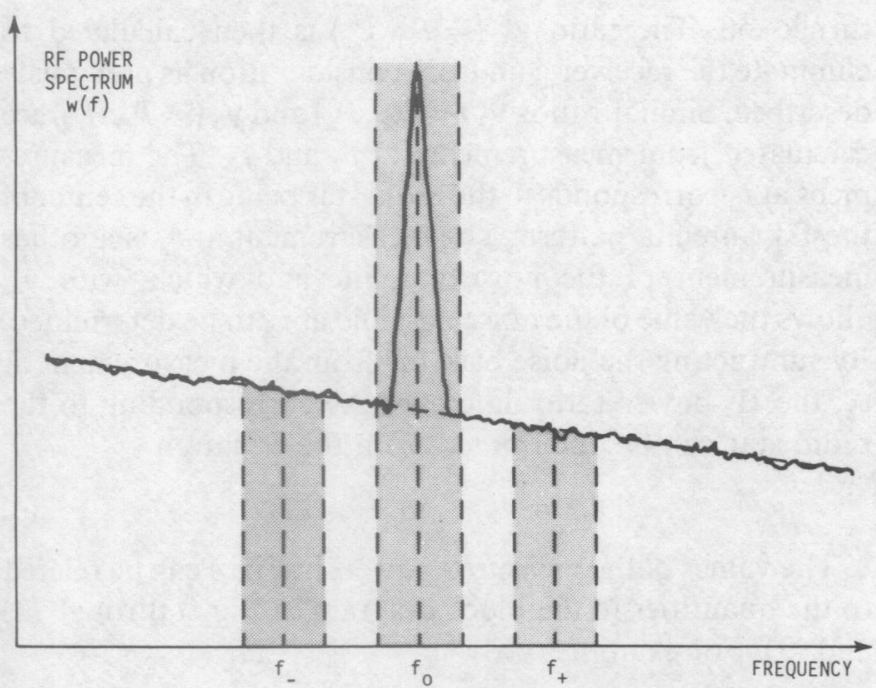

Fig. 5. A portion of the RF power spectrum as it appears at the lownoise amplifier input.

spectrum as it appears at the LNA input. In this example, the spectrum has a sloping noise baseline with the satellite signal appearing at the center frequency $f_{0}$. The shaded areas depict those frequencies passed by the limiting IF bandpass filter in the ETMS when the ET downconverter is tuned to $f_{-}, f_{0}$, or $f_{+}$, respectively. The corresponding IF powers $P_{-}$, $P_{0}$, and $P_{+}$detected by the ETMS are related to this IF power spectrum by the equation

$$
P_{i}=\int w(f) g_{i}(f) d f, \quad i=-, 0,+
$$

where $i$ indicates that the downconverter has been tuned to $f_{-}, f_{0}$, or $f_{+}$, respectively.

The procedure for normalizing these powers is different from the normalization procedure used in the $T_{a} / G$ calibration, but is still done for the same purpose, to eliminate gain fluctuations. As previously discussed in the $T_{a} / G$ measurement with the radio star $y_{1}, y_{2}$, and $y_{3}$ are calculated from pairs of power measurements with one member of each pair having the solid-state noise source turned on. With reference to Fig. 4, the magnitude of the added noise power $P_{a}$ was large enough compared to the $P_{i}(i=1,2,3)$ that when $P_{i}$ was subtracted from the measured power $P_{i}+P_{a}$ to obtain $P_{a}$, significant figures were not lost. However, in the measurement of satellite power (Fig. 5), $P_{0}$ and sometimes $P_{-}$ and $P_{+}$are sufficiently large compared to $P_{a}$ to cause significant variation in the $P_{a}$ determined from subtracting $P_{i}(i=-, 0,+)$ from $P_{i}+P_{a}$. This problem is circumvented by pointing the ET antenna away (in azimuth) from the satellite to obtain $P_{a}$ (at $f_{-}, f_{0}$, and $f_{+}$) by measuring a noise baseline comparable in magnitude to the noise baseline in Fig. 4, and then normalizing $P_{i}(i=-, 0,+)$ with the resulting $P_{a}$ 's. These $P_{a}$ 's are related to $T_{a}(f)$ and the receiver gain $g(f)$ by the equation

$$
P_{a i}=\int k T_{a}(f) g_{i}(f) d f, \quad i=-, 0,+
$$

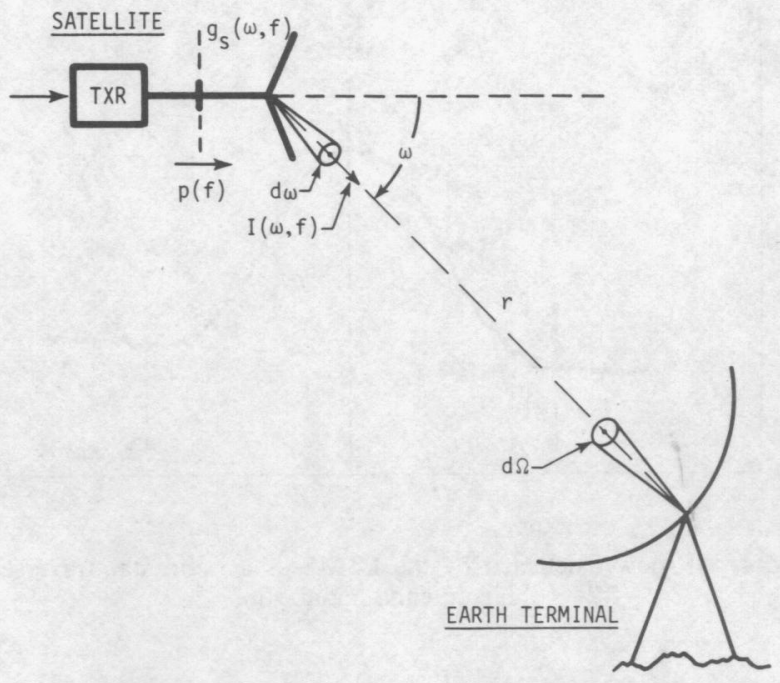

Fig. 6. Parameters used in the definition of satellite EIRP and the resulting power flux at the ET antenna aperture.

where the subscript $i$ signifies whether the ET downconverter has been tuned to $f_{-}, f_{0}$, or $f_{+}$.

The ratio difference $\Delta Y$ of the received satellite signal at the LNA input is calculated from the equation

$$
\Delta Y=y_{0}-\left(y_{-}+y_{+}\right) / 2 .
$$

With the $\Delta y$ 's of this equation replaced by their theoretical counterparts, (1) can be derived. Like the $k_{i}$ 's of (7), the $e_{i}$ 's are defined in such a manner as to obtain the functional form of (1). As in the $T_{a} / G$ measurement, the utility of the radio-star method for measuring EIRP depends upon the $e_{i}$ 's being close to unity. The working equations for the $e_{i}^{\text {'s }}$ used in the ETMS are to be found in [12].

In obtaining (1) from (10), a brief explanation of how the aspect angle correction $A$ is defined in this analysis is helpful. Fig. 6 depicts a satellite signal being radiated to an earth terminal located in the direction of the spherical angles represented by $\omega$ from the center of the mainbeam of the satellite antenna. The spectral power $p(f)$ of the signal available at the output port of the satellite transmitter (TXR) is fed to the satellite antenna whose gain in the $\omega$ direction is $g(\omega, f)$. The resulting intensity $I(\omega, f)$ radiated into the infinitesimal solid angle $d \omega$ is

$$
I(\omega, f)=p(f) g(\omega, f) .
$$

The aspect angle correction $A$ is then defined here in terms of this radiated intensity by the equation

$$
A \equiv \frac{\int I(\omega, f) d f}{\int I(0, f) d f}
$$

where $I(0, f)$ is the intensity radiated in the boresight direction of the satellite antenna, and the integrals are evaluated across a narrow bandwidth centered on the center frequency $f_{0}$ of the signal. The denominator of (12) is the EIRP. The ratio in (12) reduces with negligible error to the normalized power pattern of the satellite antenna at the center frequency $f_{0}$. 
TABLE I

Error in Measuring EIRP at $7.55 \mathrm{GHz}$ and $12^{\circ}$ Elevation Angle

\begin{tabular}{|c|c|c|}
\hline SOURCE OF ERROR & $\begin{array}{l}\text { SOURCE MAGNITUDE } \\
\text { AND UNCERTAINTY }\end{array}$ & $\begin{array}{l}\text { RESULTING PERCENT } \\
\text { ERROR IN EIRP }\end{array}$ \\
\hline 1. $\mathrm{Y}$-Factor Ratio & $10 \pm 0.029 \mathrm{~dB}$ & 0.66 \\
\hline 2. Cassiopeia A Flux Density & $586 \pm 26 \mathrm{fu}$ & 4.46 \\
\hline 3. Space Loss $(4 \pi r / \lambda)^{2}$ & $202 \pm 0.006 \mathrm{~dB}$ & 0.14 \\
\hline 4. Noise Bandwidth & $5 \mathrm{MHZ} \pm 19 \mathrm{kHz}$ & 0.38 \\
\hline 5. Atmospheric Transmission & $1 \pm 0.0702$ & 7.02 \\
\hline 6. Star Shape & $0.899 \pm 0.0070$ & 0.81 \\
\hline 7. Component Frequency Dependence & $1 \pm 0.0073$ & 0.73 \\
\hline 8. Downlink Noise Temperature Variation & $1 \pm 0.0001$ & 0.01 \\
\hline 9. ET Antenna Pointing & $1 \pm 0.0131$ & 1.31 \\
\hline 10. Polarization Mismatch & $1 \pm 0.0056$ & 0.56 \\
\hline 11. System Time Response $\& \mathrm{~T}_{\mathrm{a}}$ Instability & $1 \pm 0.0023$ & 0.23 \\
\hline 12. Aspect Angle Correction & $0 \pm 0.25 \mathrm{~dB}$ & 5.29 \\
\hline 13. Frequency & $7.55 \pm 0.00 \mathrm{GHz}$ & 0.00 \\
\hline QUADRATURE SUM OF SYSTEMATIC ERRORS & & $10.1 \%$ \\
\hline 14. Random Error From $\mathrm{T}_{\mathrm{a}} / \mathrm{G}$ Measurement & $-33.2 \pm 0.28 \mathrm{~dB}(3 \sigma)$ & 6.56 \\
\hline \multirow[t]{2}{*}{ 15. Random Error From Received Satellite Power } & $\pm 0.57 \mathrm{~dB}(3 \sigma)$ & 14.10 \\
\hline & & $18.5 \%$ \\
\hline
\end{tabular}

\section{ERror ANALYSIS}

Examination of (1) and (7) reveals that there are twentyone possible sources of systematic EIRP error. However, since $\Delta y$ and $\Delta Y$, and the $e_{i}$ 's and $k_{i}$ 's are related, the number of sources reduces to thirteen. These thirteen sources of error are listed in the first column of Table I. This table was prepared for an ET operating at $7.55 \mathrm{GHz}$ with an $18-\mathrm{m}$ antenna looking at a synchronous satellite at $12^{\circ}$ elevation above the horizon. The source magnitudes and uncertainties listed in column two reflect this choice. The error in EIRP resulting from the various uncertainties are listed in the third column. Typical random errors generated during $T_{a} / G$ and received satellite power measurements are given in rows 14 and 15 .

The $Y$-factor ratio uncertainty $(0.029 \mathrm{~dB})$ is calculated from the ratio of $y$ appearing in (4) to the $\Delta Y$ appearing in (10), although the 10-dB magnitude applies to $Y$ only. The uncertainty is mainly due to calibration errors in the IF attenuators in the ETMS, the errors due to the power bridge being considerably smaller.

The Cassiopeia A flux-density uncertainty (26 fu) (fu stands for flux units, one flux unit being equivalent to $10^{-26}$ $\mathrm{W} \mathrm{m}^{2} / \mathrm{Hz} /$ steradian) represents a careful but not quite state-of-the-art flux density calibration [9]. This uncertainty and the corresponding 4.46-percent EIRP error appears difficult to reduce at the present time.

The $0.006-\mathrm{dB}$ uncertainty in the space loss is due to range ( $r$ of Fig. 6) uncertainty which was assumed to be the daily range variation of the satellite used in the example.

The composite ET receiving system-ETMS noise bandwidth is determined at each ET from previously measurement parameters of the ETMS filters and the gain slope of the ET passband.

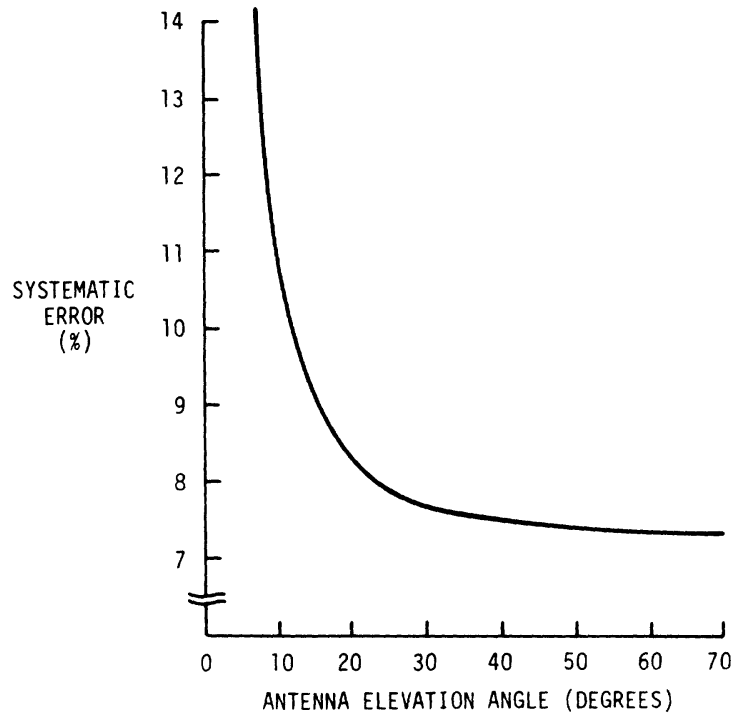

Fig. 7. Total quadrature systematic EIRP error at $7.55 \mathrm{GHz}$ versus ET antenna elevation angle.

The uncertainties listed in rows 5 through 11 were calculated from the $k_{i} / e_{i}$ ratios for $i$ equal to $1-7$, respectively. Most of the ratios shown in column 2 are unity because like correction factors tend to cancel each other. The exception is the star-shape correction factor ratio $(0.899)$ where $e_{2}$ is always unity because the satellite subtends a vanishingly small solid angle at the ET antenna, and the radio star does not.

The atmospheric transmission error $(7.02$ percent $)$ varies with elevation angle, giving the total EIRP error a characteristic shape shown in Fig. 7. Table I is a breakdown of this curve at $12^{\circ}$ elevation angle. Uncertainties in the $k_{1} / e_{1}$ ratio arise mainly from unpredictable variation in the atmo- 
spheric temperature, pressure, and water-vapor density profiles between the $T_{a} / G$ and received satellite power measurements. The large magnitude of this error $(7.02$ percent) is due in great part to a lack of measurement data and analysis concerning the tropospheric scattering effect [6], [8]. The error could probably be reduced with even a modest effort in this area. The basis for errors 6 and 7 have been previously discussed [6].

The error due to downlink noise temperature variations includes uncertainties arising from cosmic background variation around the calibrated radio star, and from portions of the satellite signal (if the signal carrier is modulated) appearing in the $f_{-}$and $f_{+}$frequency bands (see Fig. 5) reserved for the baseline noise measurements. The satellite signal was assumed to be unmodulated for these calculations, accounting for the small 0.01-percent error.

The 1.31-percent error from antenna pointing inaccuracies is essentially twice the value when pointing at either the radio star or satellite, and is calculated using an assumed pointing uncertainty of 5 percent of the antenna half-power-beamwidth.

The polarization mismatch error was calculated assuming axial ratios for the ET and satellite antennas of 0.5 and 0.05 $\mathrm{dB}$, respectively.

The time constant of the ETMS is sufficiently short and causes no appreciable EIRP error. The 0.23 -percent error in row 11 is due entirely to the $T_{a}$ instability.

The normalized power pattern of the satellite transmit antenna is needed to determine the aspect angle correction. The 5.29-percent EIRP error is the result of an aspect angle uncertainty obtained from the literature [1]. The application of emerging spherical near-field scanning techniques [10], [11] to satellite antenna measurements should significantly reduce this error.

The quadrature sum of the systematic errors in Table I is 10.1 percent, and is dominated by errors resulting from uncertainties in the Cassiopeia A flux density, the atmospheric transmission factor, and the aspect angle correction. Representative values for the random errors generated during the measurement of $T_{a} / G$ and received satellite power increase this quadrature error to 18.5 percent.

Further information concerning details of the measurement and error equations is available [12], [13].

\section{CONClusions}

The measurement of EIRP as performed by the ETMS has been described, and an outline of the derivation of the equations leading to the error analysis presented. The results of this analysis are given in Table I and Fig. 7.
The results show a 10-percent quadrature systematic EIRP error for a $12^{\circ}$ elevation angle, increasing to 19 percent $(3 \sigma)$ with addition of the random errors generated during the measurement.

At $12^{\circ}$ elevation angle, the total systematic error is dominated by errors resulting from uncertainties in the radio-star flux density, the atmospheric transmission factors, and the aspect angle correction. It appears that the latter two uncertainties might be significantly reduced with presently available knowledge and measurement techniques.

\section{ACKNOWLEDGMENT}

The author would like to thank D. Bathker and A. Freiley of the Jet Propulsion Laboratories, and T. Bremer, M. Kanda, D. Wait, and J. Wakefield of the NBS Laboratories for a number of helpful discussions.

\section{REFERENCES}

[1] E. E. Steinbrecher and L. F. Gray, "A computer controlled satellite signal monitoring system," COMSAT Tech. Rev., vol. 1, no. 1, Fall 1971.

[2] C. T. Stelzried, "The JPL total power and noise adding radiometers," NBS High Frequency and Microwave Noise Seminar, May 1970, unpublished.

[3] N. T. Larsen and F. R. Clague, "The NBS type-II power measurement system," in Proc. 1970 ISA Annu. Conf., Philadelphia, PA, Paper No. 712-70, vol. 25, Part 3, Oct. 1970.

[4] M. Kanda, "An improved solid state noise source," MTT Symp. Dig., Cherryhill, NJ, June 1976.

[5] Satellite Communications Reference Data Handbook, Defence Communications Agency, July 1972.

[6] W. C. Daywitt, "Error equations used in the NBS precision G/T measurement system," Nat. Bureau Standards Internal Note 76-842, Sept. 1976.

[7] J. D. Kraus, Radio Astronomy. New York: McGraw-Hill, 1966.

[8] H. Yokoi, M. Yamada, and T. Satoh, "Atmospheric attenuation and scintillation of microwaves from outer space," Astron. Soc. Japan, vol. 22 , no. 4 , p. $511,1970$.

[9] M. Kanda, "An error analysis for absolute flux density measurements of Cassiopeia A," IEEE Trans. Instrum. Meas., vol. IM-25, no. 3, p. 173, Sept. 1976.

[10] P. F. Wacker and A. H. Newell, "Advantages and disadvantages of planar, circular cylindrical, and spherical scanning and description of the NBS antenna scanning facilities," presented at the Workshop on Antenna Testing Techniques, European Space Research and Technology Center of the European Space Agency, Noordwijk, The Netherlands, June 6-8, 1977.

[11] F. H. Larsen, "Probe correction of spherical near-field measurements of a satellite model," presented at the Workshop on Antenna Testing Techniques, European Space Research and Technology Center of the European Space Agency, Noordwijk, The Netherlands, June 6-8, 1977.

[12] W. C. Daywitt, "Error equations used in the NBS earth terminal measurement system," NBSIR 78-869, Dec. 1977.

[13] _- "Atmospheric propagation equation used in the NBS earth terminal measurement system," NBSIR 78-883, Apr. 1978. 